\title{
Impact of Quantum Fluctuations on the Modulational Instability of a Modified Gross-Pitaevskii Equation with Two-Body Interaction
}

\author{
Camus Gaston Latchio Tiofack ${ }^{1}$, Thierry Blanchard Ekogo ${ }^{2}$, Hermance Moussambi ${ }^{3}$, \\ Alidou Mohamadou, ${ }^{4,5}$, Timoleon C. Kofane ${ }^{1}$ \\ ${ }^{1}$ Laboratory of Mechanics, Department of Physics, Faculty of Science, University of Yaounde I, Yaounde, Cameroon \\ ${ }^{2}$ Departement de Physique, Universite des Sciences et Techniques de Masuku, Franceville, Gabon \\ ${ }^{3}$ Ecole Normale Superieure, Libreville, Gabon \\ ${ }^{4}$ Max Planck Institute for the Physics of Complex Systems, Dresden, Germany \\ ${ }^{5}$ Group of Nonlinear Physics and Complex Systems, Faculty of Science, University of Douala, Douala, Cameroon \\ Email: glatchio@yahoo.fr, tekogo@yahoo.fr,mohamali@pks.mpg.de, tckofane@yahoo.com
}

Received December 19, 2011; revised April 14, 2012; accepted April 21, 2012

\begin{abstract}
Modulational instability conditions for the generation of localized structures in the context of matter waves in BoseEinstein condensates are investigated analytically and numerically. The model is based on a modified Gross-Pitaevskii equation, which account for the energy dependence of the two-body scattering amplitude. It is shown that the modified term due to the quantum fluctuations modify significantly the modulational instability gain. Direct numerical simulations of the full modified Gross-Pitaevskii equation are performed, and it is found that the modulated plane wave evolves into a train of pulses, which is destroyed at longer times due to the effects of quantum fluctuations.
\end{abstract}

Keywords: Modulational Instability; Modified Gross-Pitaevskii Equation; Quantum Fluctuations

\section{Introduction}

Bose-Einstein condensates (BEC) made of ultracold atomic alkali gases have proven to be a fertile field in the last years for the study of nonlinear matter waves in recent reviews and monographs [1,2]. Mean-field theory provides a consistent framework for the modeling of the principal characteristics of condensation and elucidates the role of the interactions between the particles. A generic theoretical model widely employed involves the Gross-Pitaevskii (GP) equation, which bears the form of a nonlinear Schrödinger-type equation with a cubic nonlinearity, taking into account boson interactions, in addition to the confinement potential imposed on the BEC in a potential trap. The scattering length as, although initially taken to be positive (accounting for repulsive interactions and prescribing condensate stability), has later been sign-inverted to negative (attractive interaction) via Feshbach resonance, in appropriately designed experiments. This allowed for the prediction of BEC state instability, eventually leading to wave collapse, which is only possible in the attractive case $\left(a_{s}<0\right)$ [3]. Modulational instability (MI) is also an interesting issue in BEC [4]. MI is an indispensable mechanism for understanding pattern formation from a uniform medium. This phenomenon appears in continuum as well as in discrete models and finds its applications in many physical settings $[5,6]$. MI occurs when a constant-wave background becomes unstable to induced sinusoidal modulations under the combined effects of nonlinearity and dispersion. The slow modulation of a monochromatic plane wave in $\mathrm{BEC}$ can leads to an exponential growth of the unstable modes and eventually may results in the formation of envelope soliton train, which is described by the GP equation. Hence, it is important and worthful to study the properties of the MI in trapped BEC. A description based on the GP equation with cubic nonlinearity, however, is adequate only at low densities.

For higher densities or stronger confinement, it has become clear that a better description of atom-atom interaction will be required. For homogeneous systems, it has been demonstrated in a recent work by Cowell et al. [7] that different potentials having the same scattering length can lead to a vastly different ground-state energy. For inhomogeneous systems, recent works on two atoms in a trap have shown that the shape-independent approximation becomes less valid under strong confinement [8]. Then, a modified GP equation, which incorporates the energy dependence of the two-body scattering 
amplitude through an effective-range expansion has been derived [9]. To the best of our knowledge, MI in this system has not been investigated. The principal objet of this paper is to show analytically conditions stability for the generation of localized structures in BEC via MI and to discuss the comparison between the linear analysis of plane wave solutions and the direct numerical simulations of the full modified GP equation.

\section{Modified Gross-Pitaevskii Equations}

The dynamic behaviour of the three dimensional (3D) modified GP equation which take into account quantum fluctuations and the shape dependence on the interaction potential can be described by [9]

$$
\begin{aligned}
i \hbar \frac{\partial \psi(r, \tau)}{\partial \tau} & =-\frac{\hbar^{2}}{2 m} \nabla^{2} \psi(r, \tau)+V(r) \psi(r, \tau) \\
& +g_{3 D}|\psi(r, \tau)|^{2} \psi(r, \tau) \\
& +g_{3 D}^{\prime}|\psi(r, \tau)|^{3} \psi(r, \tau) \\
& +g_{3 D}^{\prime \prime} \nabla^{2}\left(|\psi(r, \tau)|^{2}\right) \psi(r, \tau)
\end{aligned}
$$

where $\psi \equiv \psi(r, \tau)$ denotes the BEC wave function at position $r(r \equiv(\bar{x}, \bar{y}, \bar{z}))$ and time $\tau, \nabla^{2}$ is the Laplacian, $m$ is the mass of the condensate,

$g_{3 D}=4 \pi \hbar^{2} a_{s} / m$ characterizes the two-body interactions, $g_{3 D}^{\prime}=g \frac{32}{3 \sqrt{\pi}} a_{s}^{3 / 2}$ is the correction term due to quantum fluctuation. The extra correction term $g_{3 D}^{\prime \prime}$ incorporates the shape dependence on the interaction potential. For a hard sphere potential, $g_{3 D}^{\prime \prime}=8 \pi \hbar^{2} a_{s} / 3 m$ [9]. The potential $V(r)$ confines the atoms in a trap. For harmonic trapping, $V(r)$ is given by

$$
V(r)=\frac{1}{2} m\left(\omega_{x}^{2} \bar{x}^{2}+\omega_{y}^{2} \bar{y}^{2}+\omega_{z}^{2} \bar{z}^{2}\right)
$$

where $\omega_{x}=\alpha_{x} \omega, \omega_{y}=\alpha_{y} \omega, \omega_{z}=\alpha_{z} \omega$, are the trap frequencies, $\alpha_{x}, \alpha_{y}$, and $\alpha_{z}$ are the coupling constants along the $x, y$ and $z$ axes. When the trapping in the transverse directions is stronger, the BEC is cigar-shaped and the 3D modified GP equation can be reduced to the one dimensional (1D) modified GP equation. In order to normalize the modified GP equation, we introduce the dimensionless variables

$$
a=\sqrt{\hbar / m \omega}, x=\bar{x} / a, y=\bar{y} / a, z=\bar{z} / a, t=\omega \tau / 2,
$$

and $\phi^{\prime}=\sqrt{2 a^{3} / N} \psi$. By substituting these new variables into Equation (1), we obtain

$$
\begin{aligned}
i \frac{\partial \phi^{\prime}}{\partial t} & =-\nabla^{2} \phi^{\prime}+\left(\alpha_{x} x^{2}+\alpha_{y} y^{2}+\alpha_{z} z^{2}\right) \phi^{\prime}+\bar{g}\left|\phi^{\prime}\right|^{2} \phi^{\prime} \\
& +\bar{g}\left|\phi^{\prime}\right|^{2} \phi^{\prime}+\bar{g}_{1}\left|\phi^{\prime}\right|^{3} \phi^{\prime}+\bar{g}_{2} \nabla^{2}\left(\left|\phi^{\prime}\right|^{2}\right) \phi^{\prime}
\end{aligned}
$$

where

$$
\begin{aligned}
& \bar{g}=4 \pi N \frac{a_{s}}{a}, \\
& \bar{g}_{1}=\frac{128}{3} \sqrt{\frac{\pi}{2}} \frac{a_{s}}{a} N^{3 / 2}\left(\frac{a_{s}}{a}\right)^{5 / 2}, \\
& \bar{g}_{2}=8 \pi N\left(\frac{a_{s}}{a}\right)^{3} .
\end{aligned}
$$

We focus our attention on the trapped BEC with attractive interaction. In this respect, the coefficient $\bar{g}$ is a complex number. The following physical parameters have been used in the rest of the paper $a_{s}=2.75 \mathrm{~nm}, a=1.576 \mu \mathrm{m} \quad[10]$.

We assume a separable ansatz for the solution of Equation (2) such that [11]

$$
\phi^{\prime}(x, y, z, t)=\phi(x, t) \phi_{0}(y) \phi_{0}(z) \exp \left[-i\left(\alpha_{y}+\alpha_{z}\right) t\right]
$$

with

$$
\phi_{0}(y)=\left(\frac{\left|\alpha_{y}\right|}{\pi}\right)^{1 / 4} \exp \left(-\frac{\alpha_{y} y^{2}}{2}\right)
$$

and

$$
\phi_{0}(z)=\left(\frac{\left|\alpha_{z}\right|}{\pi}\right)^{1 / 4} \exp \left(-\frac{\alpha_{z} z^{2}}{2}\right)
$$

the respective ground state wave functions in $y$ - and $z$-directions. Using this ansatz in Equation (2), multiplying by $\phi_{0}(y) \phi_{0}(z)$, integrating over $y$ and $z$, we obtain

$$
\begin{aligned}
i \frac{\partial \phi}{\partial t} & =-\frac{\partial^{2} \phi}{\partial x^{2}}+\alpha_{x} x^{2} \phi+g_{1 D}|\phi|^{2} \phi \\
& +g_{1 D}^{\prime}|\phi|^{3} \phi+g_{1 D}^{\prime \prime} \nabla^{2}\left(|\phi|^{2}\right) \phi
\end{aligned}
$$

with

$$
\begin{aligned}
& g_{1 D}=2 N \frac{a_{s}}{a} \sqrt{\alpha_{y} \alpha_{z}}\left[1-\frac{2}{3}\left(\frac{a_{s}}{a}\right)^{2}\left(\alpha_{y}+\alpha_{z}\right)\right], \\
& g_{1 D}^{\prime}=\frac{128 \sqrt{2}}{15 \pi} N^{3 / 2}\left(\frac{a_{s}}{a}\right)^{5 / 2}\left(\alpha_{y} \alpha_{z}\right)^{3 / 4} \\
& g_{1 D}^{\prime \prime}=\frac{4}{3} N\left(\frac{a_{s}}{a}\right)^{3} \sqrt{\alpha_{y} \alpha_{z}}
\end{aligned}
$$

\section{Linear Stability Analysis}

In order to investigate the MI process in the presence of harmonic trapping potential, we begin with the modified lens-type transformation of the form $[12,13]$

$$
\phi(x, t)=\frac{1}{\sqrt{l(t)}} \varphi(X, T) \exp \left[\text { if }(t) x^{2}\right],
$$


where $f(t)$ is a real function of time,

$$
\begin{aligned}
& l(t)=\mid \cos \left(2 \sqrt{\alpha_{x}} t\right), \\
& X=\frac{x}{l(t)}, T(t)=\frac{1}{2 \sqrt{\alpha_{x}}} \tan \left(2 \sqrt{\alpha_{x}} t\right), \\
& f(t)=-\frac{\sqrt{\alpha_{x}}}{2} \tan \left(2 \sqrt{\alpha_{x}} t\right) .
\end{aligned}
$$

Upon the above conditions, the equation for $\varphi(X, T)$ becomes

$$
\begin{aligned}
i \varphi_{T} & =-\varphi_{X X}+g(T)|\varphi|^{2} \varphi+g_{1}(T)|\varphi|^{3} \varphi \\
& +g_{2}(T)\left(|\varphi|_{X X}^{2}\right) \varphi,
\end{aligned}
$$

where

$$
\begin{aligned}
& g(T)=g_{1 D}\left(1+4 \alpha_{x} T^{2}\right)^{-1 / 2} g_{1}(T)=g_{1 D}^{\prime}\left(1+4 \alpha_{x} T^{2}\right)^{-1 / 4} \\
& g_{2}(T)=g_{1 D}^{\prime \prime}\left(1+4 \alpha_{x} T^{2}\right)^{1 / 2} .
\end{aligned}
$$

Now, we analyze the MI of the modified GP equation described by Equation (7). We consider the evolution of the small modulation $\delta \phi$ according to

$$
\varphi=\left(\varphi_{0}+\delta \varphi(X, T)\right) \exp \left[-i \int \Phi(T) \mathrm{d} T\right] .
$$

where $\Phi(T)$ is a nonlinear frequency shift. Substituting Equation (8) into Equation (7) and neglecting second-order terms in $\delta \phi$ and its complex conjugate $\delta \phi^{*}$, we obtain

$$
\Phi(T)=\varphi_{0}^{2}\left[g(T)+g_{1}(T) \varphi_{0}\right] .
$$

Then, the equation describing the dynamics of the perturbation take the form

$$
\begin{aligned}
i \frac{\partial \delta \varphi}{\partial T}= & -\frac{\partial^{2} \delta \varphi}{\partial X^{2}}+\Delta(T)\left(\delta \varphi+\delta \varphi^{*}\right) \\
& +g_{2}(T) \varphi_{0}^{2}\left(\frac{\partial^{2} \delta \varphi}{\partial X^{2}}+\frac{\partial^{2} \delta \varphi^{*}}{\partial X^{2}}\right),
\end{aligned}
$$

where $\Delta(T)=\varphi_{0}^{2}\left[g(T)+\frac{3}{2} g_{1}(T) \varphi_{0}\right]$.

To perform a precise analysis of Equation (10), we use the complex representation $\delta \varphi=U+i V$. Then, Equation (10) is transformed into the following two coupled equations

$$
\begin{aligned}
& \frac{\partial U}{\partial T}+\frac{\partial^{2} V}{\partial X^{2}}=0 \\
& -\frac{\partial V}{\partial T}-2 \Delta(T) U+\Delta^{\prime}(T) \frac{\partial^{2} U}{\partial X^{2}}=0
\end{aligned}
$$

where $\Delta^{\prime}(T)=1-2 g(T) \varphi_{0}^{2}$. We assume that the amplitude perturbation varies as

$$
\begin{aligned}
& U=\operatorname{Re}\left\{U_{0} \exp \left[i\left(K X-\int \Omega(T)\right)\right]\right\}, \\
& V=\operatorname{Re}\left\{V_{0} \exp \left[i\left(K X-\int \Omega(T)\right)\right]\right\},
\end{aligned}
$$

where $K$ and $\Omega$ are, the wave number and frequency of the modulation, respectively. Substituting these expressions in Equation (11), we obtain the following time-dependent nonlinear dispersion relation

$$
\Omega^{2}=K^{4}\left[\Delta^{\prime}(T)+2 \frac{\Delta(T)}{K^{2}}\right] .
$$

If $\Omega^{2}>0$, the system is stable under modulation, otherwise if $\Omega^{2}<0$, the system is unstable. Therefore, the instability growth rate can be written as

$$
g=|\operatorname{Im}(\Omega)| .
$$

For $g_{1}=g_{2}=0$, we recover the GP equation with two-body interaction whose MI properties have been analyzed in detail by $\mathrm{Xu}$ [14]. Next, we are interested in MI conditions in BEC taking the modified GP equation as model equation and discuss the following two cases.

For a first case, we set $g_{1}=0$ (absence of quantum fluctuations) and $g_{2} \neq 0$. In this situation, the MI may develop during the time scale $t<t_{c}$, where

$$
t_{c}=\frac{1}{2 \sqrt{\alpha_{x}}} \arccos \left[\frac{1-\sqrt{1-4 A_{1} B_{1}}}{2 A_{1}}\right],
$$

with

$$
\begin{aligned}
& A_{1}=-4\left(\frac{\varphi_{0}}{K}\right)^{2} N\left(\frac{a_{s}}{a}\right) \sqrt{\alpha_{y} \alpha_{z}}\left(1-\frac{2}{3}\left(\frac{a_{s}}{a}\right)^{2}\left(\alpha_{y}+\alpha_{z}\right)\right), \\
& B_{1}=\frac{8 N}{3}\left(\frac{a_{s}}{a}\right)^{3} \varphi_{0}^{2} \sqrt{\alpha_{y} \alpha_{z}} .
\end{aligned}
$$

From Equation (14), one can see that the external trapping parameter $\alpha_{x}$ enhances the instability which occurs at short time scale for stronger trapping. Figure 1 portrays the MI gain provided by Equation (11), as a function of $K$, for three values of the trapping parameter $\alpha_{x}$. When $\alpha_{x}$ increases, both the peak gain and the MI bandwidth decrease. We have also observed that the parameter $g_{2}$ essentially does not influence the MI peak gain and the MI bandwidth.

For the second case, the effects of quantum fluctuations have been taken into account $\left(g_{1} \neq 0\right.$ and $\left.g_{2} \neq 0\right)$. In this case, it has been extremely difficult to perform a complete analytical analysis to obtain the MI critical time. However, as can be seen from Figure 2, the parameter $g_{1}$ extends the bandwidth of MI to the whole spectrum of the wave number $K$, contrary to the previous case where the instability is of the finite bandwidth type. This is one of the main result of present paper. 


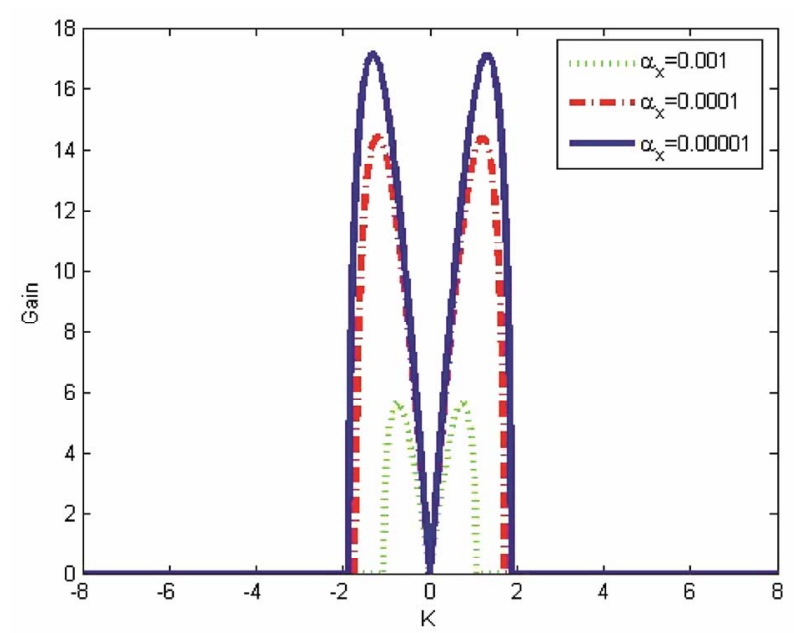

Figure 1. The MI gain in the modified GP equation for $g_{1}=$ 0 , and $g_{2} \neq 0$. The parameters used are $\varphi_{0}=10, N=50, \alpha_{y}$ $=1, \alpha_{z}=1, t=30, \alpha_{s}=-2.75 \mathrm{~nm}, \alpha=1.576 \mu \mathrm{m}$.

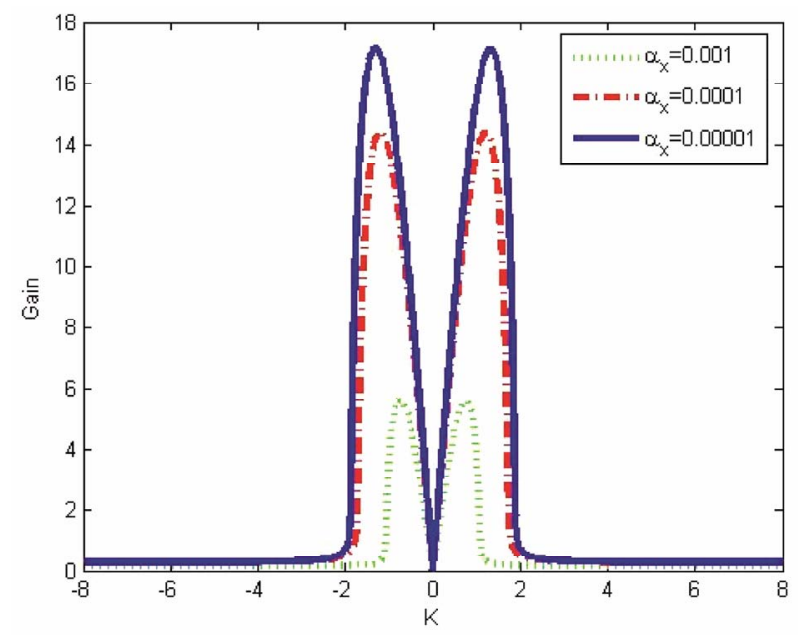

Figure 2. The MI gain in the modified GP equation for $g_{1}$ $\neq 0$, and $g_{2} \neq 0$. Other parameters are $\varphi_{0}=10, N=50, \alpha_{y}$ $=1, \alpha_{z}=1, t=30, \alpha_{s}=-2.75 \mathrm{~nm}, \alpha=1.576 \mu \mathrm{m}$.

\section{Numerical Simulations}

To verify our results and get more insight on the development of the instability, we solve numerically Equation (4) by using the split-step Fourier method with periodic boundary conditions on the spatial domain. The initial condition used is

$$
\phi(0, x)=\phi_{T F}[1+\varepsilon \cos (K x)],
$$

where $\phi_{T F}=\sqrt{\max [0 ; 1-V(x)]}$ is the background wave function in the Thomas-Fermi approximation. In a region close to the center $x=0$ and for convenient values of the potential, $\phi_{T F} \simeq 1-\frac{1}{2} \alpha_{x} x^{2} \quad[13,14]$. In all numerical simulations, we set $\varepsilon=0.001$ (small compared to $\left.\varphi_{0}\right)$. To illustrate our theoretical predictions, we con- sider two special cases. We first consider the case $g_{1}=0$ and $g_{2} \neq 0$. Figure 3 depicts the spatial evolution of a modulated amplitude of the wave for the wave number $K=0.15$. The initial wave breaks up into a pulse chain [Figure 3(a) at $t=20$ and Figure 3(b) at $t=50]$ as the time increases.

Figure 4 shows the time evolution of the maximal amplitude in Figures 4(a) and (c); and the spatiotemporal evolution of the modulated envelope $|\phi|^{2}$ in Figures 4(b) and (d). By comparing Figures 4(a) and (b), we realize that for the strong trapping case in Figure 4(a), the rate of MI increases. Secondly, we consider the general modified GP equation with $g_{1} \neq 0$, and $g_{2} \neq 0$. From Figure 2, we choose $K=0.15$, which belongs to the MI zone. Figure 5 portrays the time evolution of the maximal amplitude in Figures 5(a) and (c); and the spatiotemporal evolution of $|\phi|^{2}$ in Figures 5(b) and (d). We obtain also a soliton wave train, formed as a result of the instability. However, by comparing Figure 5 and Figure 4 obtained in the previous case, we realize that the quantum fluctuations destroyed the wave trains, which is also one of the main findings of this work.

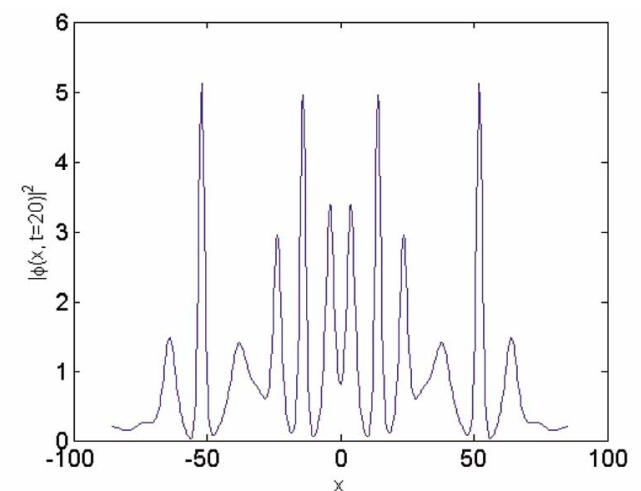

(a)

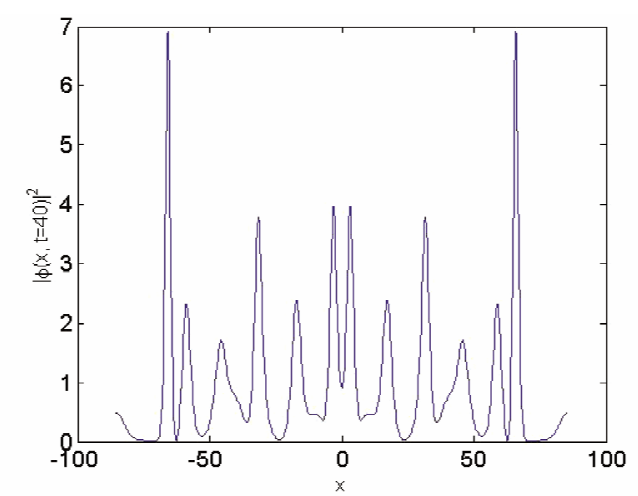

(b)

Figure 3. Spatial evolution of the modulated waves in terms of solitary waves on top of the Thomas-Fermi cloud, introducing the occurrence of the modulational instability through the system. (a) $t=20$; (b) $t=40$. The other parameters used are $\alpha_{x}=0.0004, \alpha_{y}=1, \alpha_{z}=1, K=0.15, \varepsilon=$ $0.001, N=20, \alpha_{s}=-2.75 \mathrm{~nm}, \alpha=1.576 \mu \mathrm{m}$. 


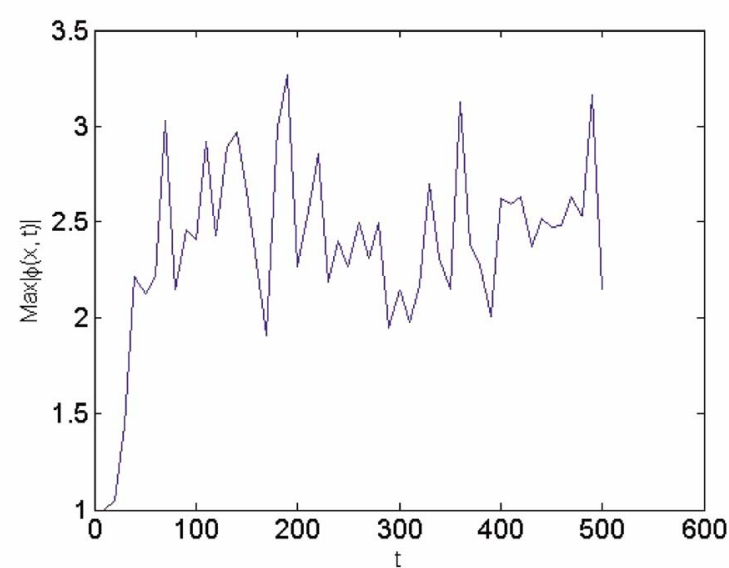

(a)

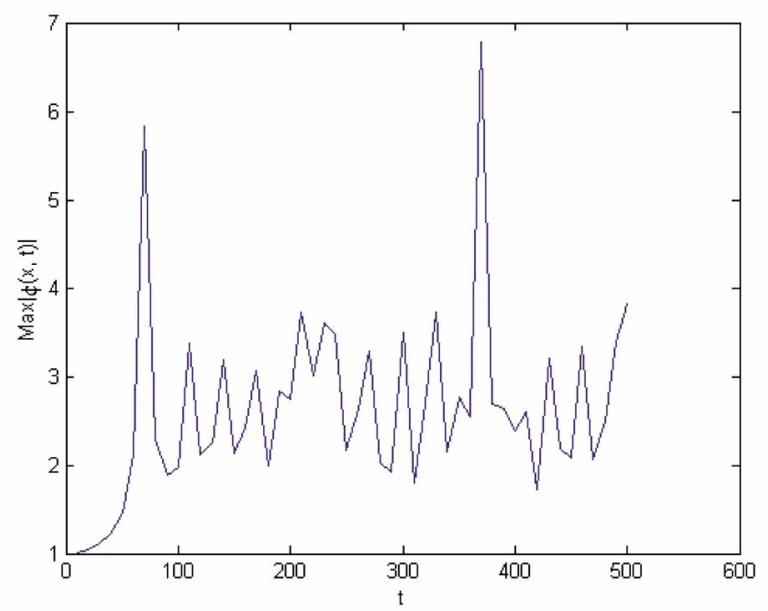

(c)

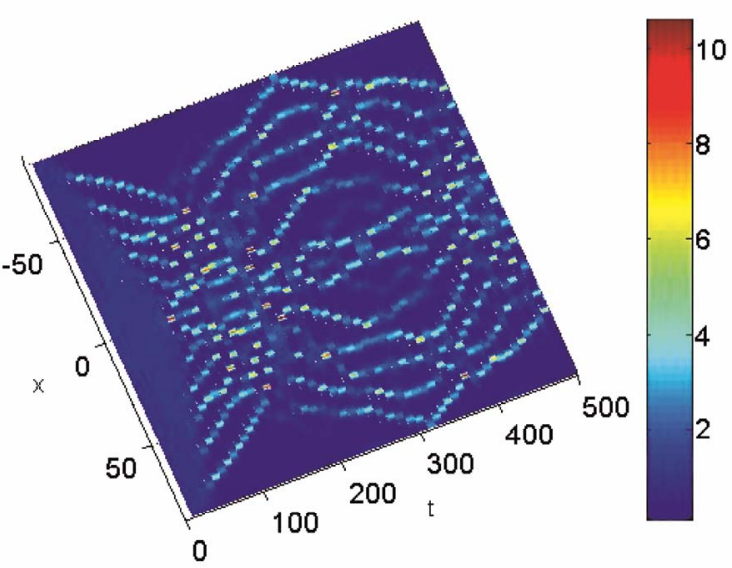

(b)

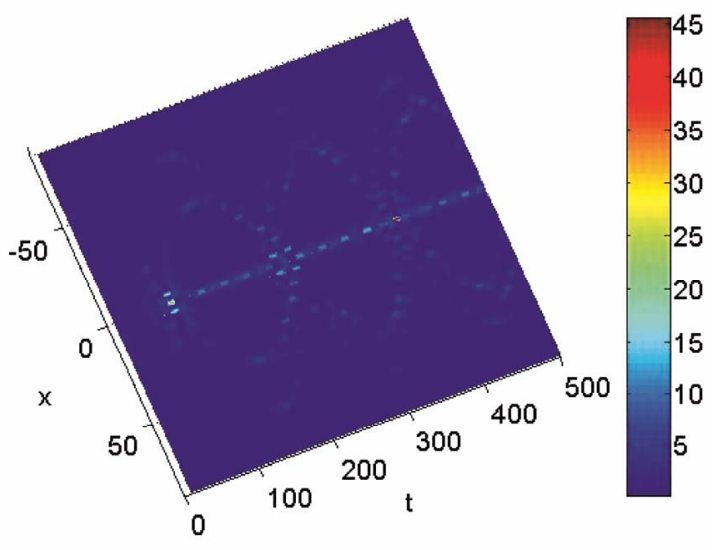

(d)

Figure 4. Spatiotemporal evolution of the maximum amplitude of the waves showing the formation of wave trains in the case $g_{1}=0$, and $g_{2} \neq 0$. The parameters used are $K=0.15, \varepsilon=0.001, \alpha_{y}=1, \alpha_{z}=1, \alpha_{s}=-2.75 \mathrm{~nm}, \alpha=1.576 \mu \mathrm{m}$. (a) $\max |\phi|$ for $N$ $=50, \alpha_{x}=0.0004$ (weak trapping); (b) $|\phi(x, t)|^{2}$ for $N=50, \alpha_{x}=0.0004$ (weak trapping); (c) $\max |\phi|$ for $N=20, \alpha_{x}=0.0004$ (strong trapping); (d) $|\phi(x, t)|^{2}$ for $N=20, \alpha_{x}=0.0004$ (strong trapping).

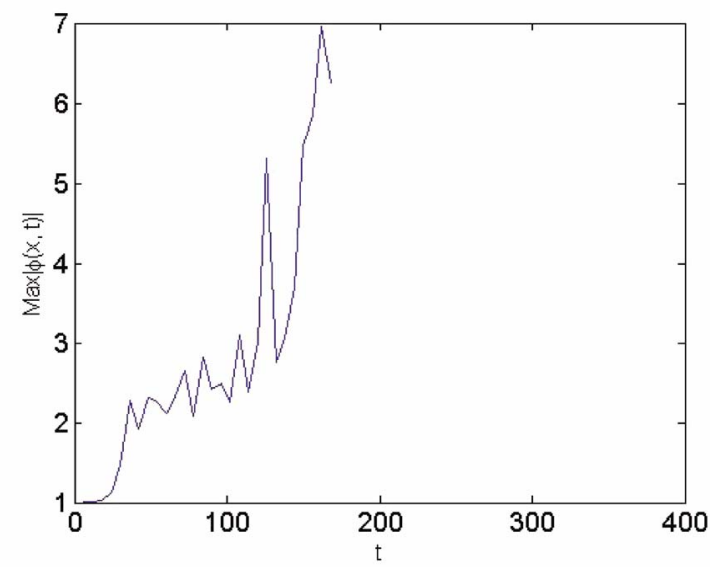

(a)

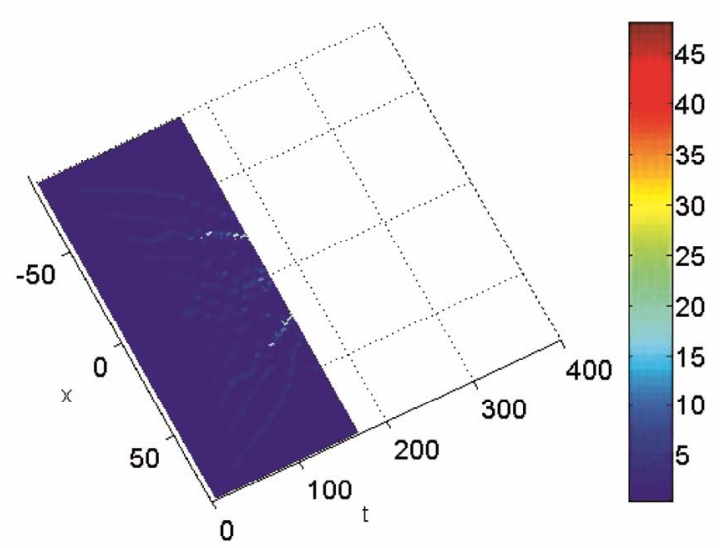

(b) 


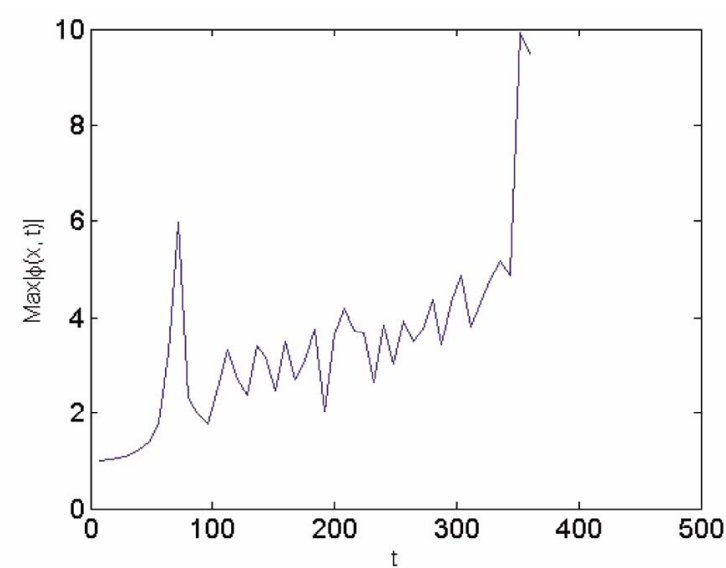

(c)

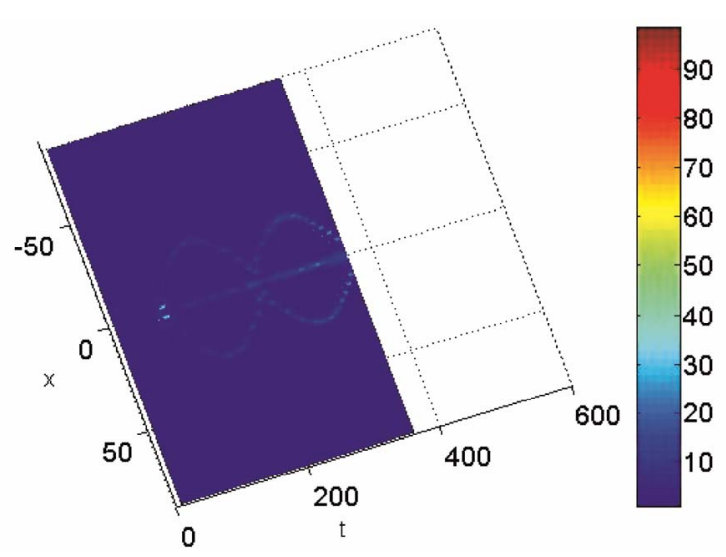

(d)

Figure 5. Spatiotemporal evolution of the maximum amplitude of the waves showing the formation of wave trains in the case $g_{1}=0$, and $g_{2} \neq 0$. The parameters used are $K=0.15, \varepsilon=0.001, \alpha_{y}=1, \alpha_{z}=1, \alpha_{s}=-2.75 \mathrm{~nm}, \alpha=1.576 \mu \mathrm{m}$. (a) $\max |\phi|$ for $N$ $=50, \alpha_{x}=0.0004$ (weak trapping); (b) $|\phi(x, t)|^{2}$ for $N=50, \alpha_{x}=0.0004$, (weak trapping); (c) $\max |\phi|$ for $N=\mathbf{2 0}, \alpha_{x}=0.0004$ (strong trapping); (d) $|\phi(x, t)|^{2}$ for $N=20, \alpha_{x}=0.0004$ (strong trapping).

\section{Conclusion}

In summary, we have investigated analytically and numerically the MI in the modified GP equation. By using a theoretical model which includes the energy dependence of the two-body scattering amplitude, our results show that MI in the modified GP equation is qualitatively different. By taking the modified terms as $g_{1}=0$ and $g_{2} \neq 0$, a new explicit time-dependent criteria for MI has been obtained. Moreover, both the MI gain peak and bandwidth decrease with the trapping of the condensate. In the case $g_{1} \neq 0$ and $g_{2} \neq 0$, it is shown that the quantum fluctuations extend the $\mathrm{MI}$ gain in the whole spectrum of the wave number $K$. By numerical simulations, we have observed that when the quantum fluctuations are not introduced in the system, wave patterns propagate in the medium without vanishing. When the quantum fluctuations are introduced, the wave trains are destroyed at long time. These wave trains can be exploited for the realization of laser sources adapted to ultrahigh bit-rate optical transmissions. It open also the possibilities for future applications in coherent atom optics, atom interferometry and atom transport.

\section{REFERENCES}

[1] C. Pethick and H. Smith, "Bose-Einstein Condensation in Dilute Gases," Cambridge University Press, Cambridge, 2003.

[2] L. Pitaevskii and S. Stringari, "Bose-Einstein Condensation in Dilute Gases," Oxford University Press, New York, 2003.

[3] J. M. Gerton, D. Strekalov, I. Prodan and R. G. Hulet,
"Direct Observation of Growth and Collapse of a BoseEinstein Condensate with Attractive Interaction," Nature, Vol. 408, 2000, pp. 692-695. doi:10.1038/35047030B

[4] T. S. Raju, P. K. Panigrahi and K. Porsezian, "Modulational Instability of Two-Component Bose-Einstein Condensates in a Quasi-One Dimensional Geometry," Physics Review A, Vol. 71, No. 3, 2005, Article ID: 035601. doi:10.1103/PhysRevA.71.035601

[5] A. Hasegawa and F. Tappert, "Transmission of Stationary Nonlinear Optical Pulses in Dispersive Dielectric Fibers," Applied Physics Letter, Vol. 23, No. 3, 1973, pp. 142-144. doi:10.1063/1.1654836

[6] C. Tabi, A. Mohamadou and T. C. Kofane, "Modulated Wave Packets in DNA and Impact of Viscosity," Chinese Physics Letter, Vol. 26, No. 6, 2009, Article ID: 068703. doi:10.1088/0256-307X/26/6/068703

[7] S. Cowell, H. Heiselberg, I. E. Mazets, J. Morales, V. R. Pandhari-Pande and C. J. Pethick, "Cold Bose Gases with Large Scattering Lengths," Physics Review Letter, Vol. 88, 2002, pp. 210403-210406. doi:10.1103/PhysRevLett.88.210403

[8] E. Tiesinga, C. J. Williams, F. H. Mies and P. S. Julienne, "Interacting Atoms under Strong Quantum Confinement," Physics Review A, Vol. 61, No. 6, 2000, Article ID: 063416. doi:10.1103/PhysRevA.61.063416

[9] H. Fu, Y. Wang and B. Gao, "Beyond the Fermi Pseudopotential: A Modified Gross-Pitaevskii Equation," Physics Review A, Vol. 67, No. 5, 2003, Article ID: 053612. doi:10.1103/PhysRevA.67.053612

[10] G. Theocharis, Z. Rapti, P. G. Kevrekidis, D. J. Frantzeskakis and V. V. Konotop, "Modulational Instability of Gross-Pitaevskii-Type Equations in 1+1 Dimensions," Physics Review A, Vol. 67, No. 6, 2003, Article ID: 063610. doi:10.1103/PhysRevA.67.063610

[11] P. Muruganandam and S. Adhikari, "Fortran Programs 
for the Time-Dependent Gross-Pitaevskii Equation in a Fully Anisotropic Trap," Computer Physics Communication, Vol. 180, No. 10, 2009, pp. 1888-1912. doi:10.1016/j.cpc.2009.04.015

[12] L.Wu and J. F. Zhang, "Modulational Instability of (1+1)Dimensional Bose-Einstein Condensate with Three-Body Interatomic Interaction," Chinese Physics Letter, Vol. 24, No. 6, 2007, pp. 1471-1474. doi:10.1088/0256-307X/24/6/012
[13] E. Wamba, A. Mohamadou and T. C. Kofane, "Modulational Instability of a Trapped Bose-Einstein Condensate with Two- and Three-Body Interactions," Physics Review E, Vol. 77, No. 4, 2008, Article ID: 046216. doi:10.1103/PhysRevE.77.046216

[14] J. K. Xu, "Modulational Instability of the Trapped BoseEinstein Condensates," Physics Letter A, Vol. 341, No. 5-6, 2005, pp. 527-531. doi:10.1016/i.physleta.2005.05.018 